\title{
The multiple roles of the Mre11 complex for meiotic recombination
}

\author{
Valérie Borde* \\ Institut Curie, Recombinaison et Instabilité Génétique, Centre de Recherche, UMR7147 CNRS-Institut \\ Curie-Université P. et M. Curie, 26 rue d'Ulm, 75248, Paris Cedex 05,France; E-mail: valerie.borde@curie.fr \\ * Correspondence
}

Key words: DNA damage checkpoint, double-strand breaks, meiosis, Mre11, Saccharomyces cerevisiae, yeast

\begin{abstract}
During the first meiotic prophase, numerous DNA double-strand breaks (DSB) are formed in the genome in order to initiate recombination between homologous chromosomes. The conserved Mre11 complex, formed of Mre11, Rad50 and Nbs1 (Xrs2 in Saccharomyces cerevisiae) proteins, plays a crucial role in mitotic cells for sensing and repairing DSB. In meiosis the Mre11 complex is also required for meiotic recombination. Depending on the organisms, the Mre11 complex is required for the formation of the DSB catalysed by the transesterase Spo11 protein. It then plays a unique function in removing covalently attached Spo11 from the $5^{\prime}$ extremity of the breaks through its nuclease activity, to allow further break resection. Finally, the Mre 11 complex also plays a role during meiosis in bridging DNA molecules together and in sensing Spo11 DSB and activating the DNA damage checkpoint. In this article the different biochemical functions of the Mre 11 complex required during meiosis are reviewed, as well as the consequences of Mre11 complex inactivation for meiosis in several organisms. Finally, I describe the meiotic phenotypes of several animal models that have been developed to model hypomorphic mutations of the Mre11 complex, involved in humans in some genetic instability disorders.
\end{abstract}

\section{Introduction}

In most sexually reproducing organisms meiotic recombination between homologous chromosomes is needed to ensure a physical connection that allows them to orient properly on the spindle and segregate accurately at the first division. Meiotic recombination is initiated by the formation of programmed DNA double-strand breaks (DSB) catalysed by Spo11, which are then repaired by homologous recombination (reviewed in Keeney 2001). The repair of meiotic DSB utilizes many of the mitotic homologous recombination proteins, but also has some unique specificities, among which a bias towards repair with the homologous chromosome rather than with the sister chromatid, and the need to remove the
Spo11 protein from DSB ends. A central player in the signalling and repair of DSB in mitotic cells is the Mre11 complex. Hypomorphic mutations in two of its components, Nbs1 and Mre11, are responsible for Nijmegen breakage syndrome (NBS) and ataxialike disorder (ATLD) respectively, two human genetic instability disorders. Not surprisingly, the Mre11 complex is also involved in the meiotic recombination pathway. This review analyses the different studies of the Mre11 complex during meiosis. First, I emphasize the biochemical properties of each member of the Mre11 complex, and detail the studies made in Saccharomyces cerevisiae using separation of function mutants, which allowed better understanding of which functions of the Mre11 complex are solicited during meiosis. Then I describe 
the evolutionary conservation of the meiotic roles of the Mre11 complex and the meiotic phenotypes of the recent animal models that have been developed for Mre11 complex deficiency.

\section{Structure and biochemical properties of the Mre11 complex}

The conserved Mre11 complex influences each aspect of chromosome break metabolism. This complex contains three proteins, Mre11, Rad50 and Nbs1 (Xrs2 in S. cerevisiae). It is involved in telomere maintenance, cell cycle responses to DNA damage and DSB repair by both homologous recombination and non-homologous end joining (reviewed in D'Amours \& Jackson 2002). Many recent studies concerning the biochemical properties and structure of the complex have shed light on how it may fulfil its various roles in DSB repair.

The Rad50 protein is a split ABC-type ATPase which contains two heptad repeats in its centre, that fold into a coiled-coil, bringing the two $\mathrm{N}$ - and C-terminal ATPase motifs Walker A and B in close proximity (de Jager et al. 2001) (Figure 1). These motifs also interact with two Mre11 monomers, forming a globular domain that interacts with DNA (de Jager et al. 2001). The apex of the Rad50 coiledcoil contains a zinc hook that may facilitate interactions between Rad50 molecules (Hopfner et al. 2002). ATP binding promotes the dimerization between two catalytical Rad50 domains, which is crucial for efficient DNA binding (Hopfner et al. 2000) and thus for all functions of the Mre11 complex (Alani et al. 1990). The association between two Rad50 protomers can either form a large loop, or can also occur between two complexes bound on two different DNA molecules. Both modes of association have been seen by atomic force and electron microscopy. Upon DNA binding via the globular domain, the intracomplex interaction is prevented and the intermolecular configuration is favoured, which allows DNA tethering (Moreno-Herrero et al. 2005). In addition to ATP binding and hydrolysis, the conserved 'signature motif' in the C terminal ATPase domain of Rad50 bears an adenylate kinase activity, which is required for efficient tethering between different DNA molecules, independently of DNA binding (Bhaskara et al. 2007). All these observations have led to the proposal that this molecular bridging by the Mre11 complex via the $\operatorname{Rad} 50$ coiled-coils constitutes the molecular basis of the Mre11 complex's influence on recombinational DNA repair.

The Mre11 protein is a DNA-binding protein that contains four conserved N-terminal phosphoesterase motifs (Figure 1). Human and budding yeast Mre11 have single-strand endonuclease activity, $3^{\prime}-5^{\prime}$ doublestrand exonuclease activity, as well as weak DNA unwinding (stem-loop opening) activity (Paull \& Gellert 1998, Usui et al. 1998, Trujillo et al. 2003). The observed 3' -5 ' polarity of exonuclease activity was at first surprising because genetic and physical analyses of meiotic recombination and mitotic DSB repair had suggested that the Mre11 complex is involved in $5^{\prime}-3^{\prime}$ exonucleolytic resection. However, hypomorphic mutations that abolish the nuclease activity of Mre11 do not impair most forms of DSB repair in mitotic cells, indicating that the complex's contribution to that process is largely independent of its nuclease activity (Tsubouchi \& Ogawa 1998, Usui et al. 1998, Bressan et al. 1999, Moreau et al. 1999, Lewis et al. 2004, Llorente \& Symington 2004, Krogh et al. 2005). Mre11 also contains two DNA-binding domains, one conserved in the centre of the protein and one less conserved in the C-terminus (Usui et al. 1998).

Whereas Mre11 and Rad50 are strongly conserved in bacteria (SbcCD), archaebacteria and eukaryotes, the third member of the complex is less well conserved among species. Nevertheless, NBS1 has a functional homologue, Xrs2, in $S$. cerevisiae. The two proteins share some common motifs: a N-terminal FHA domain, involved in protein-protein interactions and which binds to phosphorylated histone H2AX (Kobayashi et al. 2002), phosphorylation sites for the checkpoint protein Atm (Tel1 in yeast) and a conserved Cterminal domain (CCD) involved in interaction with Mre11 (Figure 1; reviewed in Kobayashi et al. 2004). Both Nbs1 and Xrs2 proteins enhance Mre11 nuclease activity in vitro (Carney et al. 1998, Paull \& Gellert 1999, Desai-Mehta et al. 2001, Tauchi et al. 2001). Mutant studies in S. cerevisiae have shown that interaction of Xrs2 with Mre11 is crucial to all the functions of the Mre11 complex since a single amino-acid mutation disrupting this interaction results in a Mre11 complex-null phenotype (Tsukamoto et al. 2005). Similarly, in NBS patients with the $657 \mathrm{del} 5$ allele, the mutated protein is in reduced amounts and $\mathrm{hMre} 11 \mathrm{p}$ is mislocalized to 

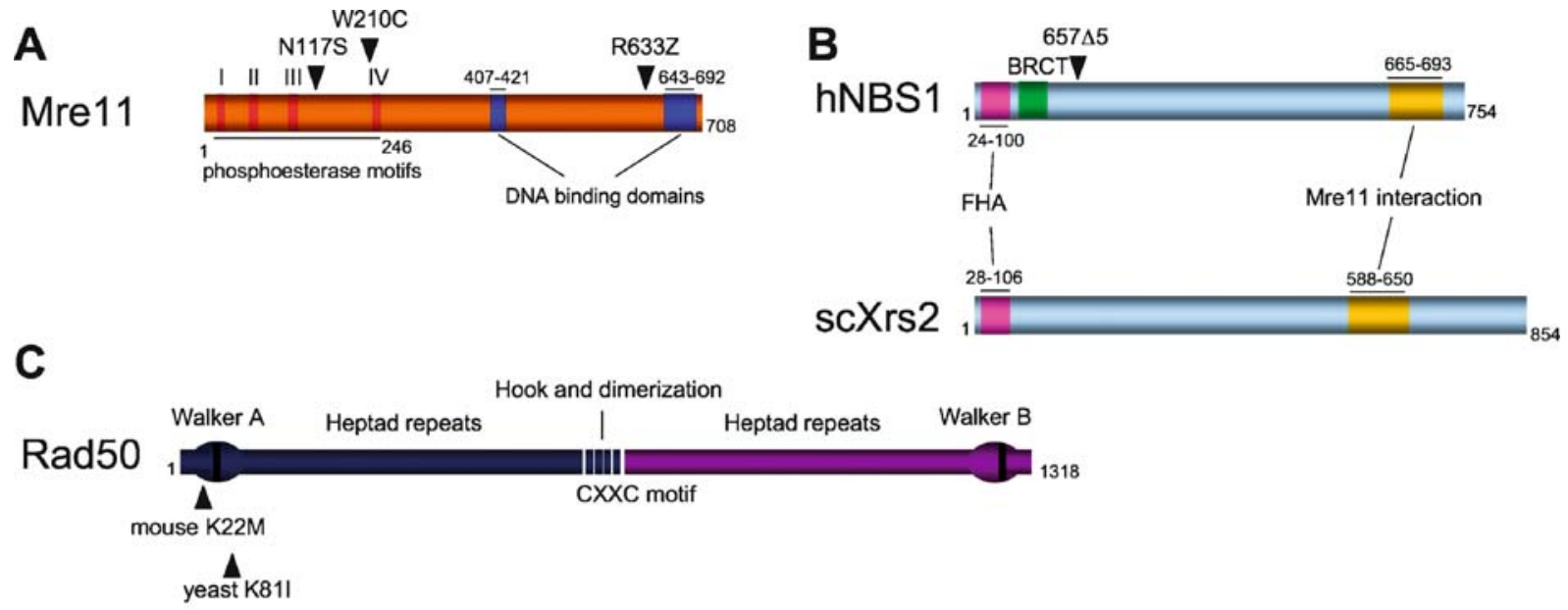

D

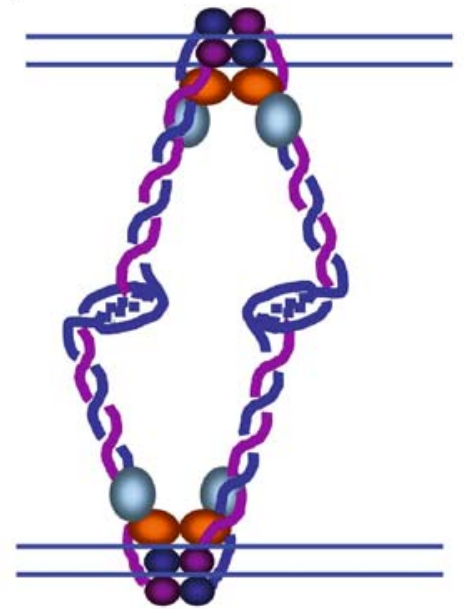

Figure 1. Structure of the Mre11 complex. A: Structure of the human Mre11 protein. The two DNA binding domains and the nuclease domain with the four conserved phosphoesterase motifs are indicated. The position of the three mutations involved in human ATLD is also shown. B: Structures of the human NBS1 and the budding yeast Xrs2 proteins. The different FHA, BRCT and Mre11 interaction domains are indicated, as well as the mutation found in most NBS patients (657de15), giving rise to a N-terminal truncated protein lacking the FHA and BRCT domains. C: Primary structure of the Rad50 protein, comprising two Walker A and B motifs carrying the ATPase activity, two heptad repeats and a central portion forming a zinc hook that facilitates interaction between Rad50 molecules (Wiltzius et al. 2005). D: Proposed structure of the Mre11 complex. The position of Nbs1/Xrs2 is speculative. Two Rad50/Mre11 dimers (each composed of Rad50 2 Mre $11_{2}$ ) interact with each other via their hooks, here tethering two DNA molecules, as deduced from electron microscopy studies (Hopfner et al. 2002).

the cytoplasm (Carney et al. 1998, Tauchi et al. 2001). One of the roles of both Nbs1 and Xrs2 might thus be to maintain an intranuclear localization of Mre11. Another role is to activate Atm autophosphorylation specifically in response to DNA damage induced by ionizing radiation (Difilippantonio et al. 2005) and thus the Mre11 complex works not only downstream but also upstream of Atm. This is consistent with findings that purified Mre11 complex can activate Atm in vitro (Lee \& Paull 2004), as well as studies in yeast and human cells that show that the Mre11 complex recruits Atm/Tel1 to sites of DNA damage (Nakada et al. 2003, Uziel et al. 2003). The yeast Xrs2 and mammalian NBS1 proteins interact with Tell and Atm, respectively, through their C terminus (Nakada et al. 2003, You et al. 2005). This interaction is specifically required for telomere elongation (Shima et al. 2005, Tsukamoto et al. 2005). The role of the FHA domain is less clear. Its interaction in mammals with phosphorylated histone H2AX (Kobayashi et al. 2002), which itself is phosphorylated through Atm, suggests that it might 
serve to recruit Nbs1 to sites of damage to amplify the checkpoint response to DSB. In yeast the FHA domain is dispensable for all the mitotic functions of the Mre11 complex (Shima et al. 2005, Tsukamoto et al. 2005).

\section{Roles of the Mre11 complex in budding yeast meiosis}

In meiosis the role of the Mre11 complex has been especially well documented in S. cerevisiae and the use of separation of function mutants of each member of the complex allowed to identify the different functions of the Mre11 complex required for meiotic recombination. The mrell mutation was first described as meiotic recombination deficient (Ajimura et al. 1993), and it was shown that deletion of either member of the Mre11 complex leads to an absence of meiotic recombination and extremely low spore viability (Alani et al. 1990, Ivanov et al. 1992). This absence is due to a lack of meiotic DSB, which are normally catalysed by the Spo11 protein (Alani et al. 1990, Johzuka \& Ogawa 1995, Shima et al. 2005). Some separation of function mutants have shown that the Mre11 complex is also needed once meiotic DSB are formed, for their resection and repair.

\section{The budding yeast complex is required for meiotic DSB formation independently of its catalytic nuclease activity}

The three members of the Mre11 complex are among the nine proteins required, in addition to the catalytic component Spo11, for the formation of meiotic DSB (reviewed in Keeney 2001). One specific region of Mre11, in the C-terminus, is strictly required for this: indeed, its deletion leads to an absence of DSB formation and the production of dead spores. In two cases, the mre11-5 mutant, truncated for the last $137 \mathrm{aa}$, and the mrel1 $1 \Delta 49$ mutant, truncated for the last $49 \mathrm{aa}$, the truncated protein was fully functional for its mitotic functions (Furuse et al. 1998, Usui et al. 1998). In another case a truncated form of Mre11 lacking the last 128aa (mrel1-T4) was deficient for DSB formation but also partially deficient for MMS tolerance (Nairz \& Klein 1997). However, whenever tested it appears that these truncated Mre11 proteins are able to resect and repair meiotic
DSB made by Spo11, since they show intragenic complementation with mrell alleles proficient in DSB formation but deficient in meiotic DSB resection (Nairz \& Klein 1997, Usui et al. 1998). Chromatin immunoprecipitation studies have shown that, in wild-type cells, Mre11 transiently associates with DSB sites, at the time of meiotic DSB formation (Borde et al. 2004). This association does not require DSB formation and still timely occurs in a strain carrying the catalytic mutant spol1Y135F allele. Surprisingly, Rad50 is not required for Mre11 association with DSB sites. Thus, the functional complex is not needed. Similarly, loading of Mre11 onto telomeres during late $\mathrm{S}$ phase also occurs normally in rad50 cells (Takata et al. 2005). In agreement with the notion that an intact Mre11 complex is not required for meiotic DSB formation is that meiotic DSB are normally formed in a mre11-58S mutant, which does not form a complex with $\operatorname{Rad} 50$ and is deficient for all the other known functions of the Mre11 complex (Usui et al. 1998). Thus, the function of Mre11 for DSB formation is probably exerted through its $\mathrm{C}$-terminal part. When assessed by chromatin immunoprecipitation, this C-terminal part is required for Mre11 association with DSB sites. The fact that all DSB proteins are required for Mre11 association with DSB sites suggests that this interaction does involve direct DNA binding but rather binding to some of these proteins (Borde et al. 2004). Consistently, the purified C-terminal part of Mre11 interacts in meiotic extracts with three meiosisspecific proteins of a molecular weight compatible with the size of Rec102, Rec104 and Mer2, three of the proteins required for DSB formation (Usui et al. 1998). These results agree well with some twohybrid results showing that the Mre11 protein interacts mitotically with Mer2 and Rec104, which itself interacts with Rec102 (Arora et al. 2004).

Xrs2 interacts by mitotic two hybrid with Mer2 (Arora et al. 2004), which indicates that it may also play a non-catalytical, maybe structural, role in the 'pre-DSB' complex. In addition, the meiotic DSB formation phenotype of $x r s 2 \Delta$ may partially be explained by the fact that Xrs2 is required for efficient nuclear localization of Mre11 (Shima et al. 2005, Tsukamoto et al. 2005). Fusing Mre11 to a nuclear localization signal bypasses the requirement for Xrs2 for some functions of the Mre11 complex. This suggests that, in $x r s 2 \Delta$ cells, there is not enough Mre11 protein in the meiotic nucleus for DSB 
formation. Indeed, Mre11 association with DSB sites requires Xrs2 (Borde et al. 2004). However, nuclear localization of Mre11 does not bypass the requirement for Xrs2 in DSB formation, and mutagenesis analyses have shown that a minimal region of 80 amino acids of Xrs2, in addition to binding to Mre11, is required for DSB formation (Tsubouchi \& Ogawa 1998). In addition, a limited N-terminal truncation of Xrs2 (the first 84 amino acids) impaired meiotic DSB formation, whereas larger truncations did not (Shima et al. 2005, Tsukamoto et al. 2005). One explanation is that these $\mathrm{N}$ terminal truncations might induce conformational changes that impair the meiotic functions of the Mre11 complex whereas more extensive truncations of Xrs2 remove such impairment (Tsukamoto et al. 2005).

For Rad50 the function required for meiotic DSB formation remains elusive. It is not required for Mre11 to interact with DSB sites (Borde et al. 2004); however, in rad50 cells Mre11 association with DSB sites is unproductive for DSB formation. In the mre11-58S mutant cells meiotic DSB are formed (Tsubouchi \& Ogawa 1998, Usui et al. 1998). In-vitro studies have shown that the interaction between this mutated Mre11 and Rad50 is abolished, which led to the suggestion that a complex formation between Mre11 and Rad50 is not required for DSB formation (Usui et al. 1998) Similarly, interaction between Mre11 and Rad50 is severely reduced in the nuclease-deficient D16A mutant, which is proficient for meiotic DSB formation (Furuse et al. 1998, Krogh et al. 2005). However, Rad50 is strictly required for DSB formation. Point mutations in the consensus motif involved in ATP binding, which is crucial for efficient DNA binding (Hopfner et al. 2000), completely abolish meiotic DSB formation (Alani et al. 1990). By a two-hybrid test, Rad50 does not interact directly with any of the 'DSB proteins' except Mre11 (Arora et al. 2004). One clue about the role of Rad50 in DSB formation came from a nice study that examined the role of the dimerization hook domain of Rad50 (Wiltzius et al. 2005). Crystallographic studies have shown that this domain allows dimerization between two Mre11 complexes to tether DNA molecules (Hopfner et al. 2002). Hook mutants of Rad50 were deficient in meiotic DSB formation, and restoration of dimerization using a conditional dimerization module restored DSB formation (Wiltzius et al. 2005). Since meiotic DSB are formed in the chromatin loops rather than on the axial elements (Zickler \& Kleckner 1999, Blat et al. 2002), a role for Rad50 might be to bridge sister chromatids in order to establish a proper architecture of the sisters engaged in the pre-DSB complex (Wiltzius et al. 2005). Finally, a recent study reported that a mutation affecting the adenylate kinase activity of Rad50, necessary for DNA tethering, also abolishes the formation of viable spores, but this study did not examine if meiotic DSB are formed or not (Bhaskara et al. 2007).

\section{Roles of the Mrell complex for resection and repair of Spoll DSB}

Meiotic DSB differ from most DSB occurring either accidentally during replication or after irradiation or drug treatment, in that they have a covalentlyattached Spo11 protein at each of their $5^{\prime}$ ends, that needs to be removed for further processing by $5^{\prime}$ to $3^{\prime}$ resection, and repair by homologous recombination. Thus, in addition to the proteins required for repairing DSB by homologous recombination, they require an additional specific enzymatic activity. The mode of processing of these DSB was recently identified by Scott Keeney's laboratory, where it was shown that removal of covalently bound Spo11 occurs by endonucleolytic cleavage a few bases away from the break site, which frees an oligonucleotide with attached Spo11 (Neale et al. 2005). The Mre11 complex is very probably responsible for this activity, both based on its nuclease biochemical activity, and on the long-known phenotype of separation of function mutants as described below. A specific class of separation of function mutants of RAD50, named rad50S, allow DSB formation but are totally defective for processing of Spo11-induced meiotic DSB, and thus accumulate during meiosis unprocessed DSB with covalently attached Spo11 protein at their $5^{\prime}$ ends (Alani et al. 1990, Keeney et al. 1997). However, they show almost wild-type resistance to clastogens, such as MMS. The deletion of the SAE2/COMI gene, of unknown biochemical function, produces exactly the same phenotypes (McKee \& Kleckner 1997, Prinz et al. 1997). Accordingly, the Spo11-oligonucleotide complexes are not observed in rad50S nor in sae $2 \Delta$ mutants (Neale et al. 2005). The mutant Rad50S protein is mutated in the conserved Walker A ATPase motif (Figure 1). How this mutation affects the in-vitro functions of the Mre11 complex has not been 
determined. On the crystal structure of Rad50, seven of the nine rad50S mutations reported (Alani et al. 1990) cluster to a narrow surface patch that forms a probable protein interaction site (Hopfner et al. 2000). Thus maybe rad50S mutations affect the interaction between Rad50 and Sae2 (Rattray et al. 2001, Usui et al. 2001). Separation of function mutants of Mre11 has also been identified, which allow meiotic DSB formation but do not process DSB (Nairz \& Klein 1997, Tsubouchi \& Ogawa 1998, Furuse et al. 1998, Moreau et al. 1999). Mutations lie in the $\mathrm{N}$ terminal part of the protein, containing the conserved phosphoesterase motifs. When examined, the mutants showing this phenotype lacked nuclease activity in vitro (mutants D16A, D56N, H125N, H213Y (mrel1-58) and (mrel1-6)), demonstrating that the nuclease activity of Mre11 is probably the activity required for Spo11 removal and end resection (Furuse et al. 1998, Usui et al. 1998, Moreau et al. 1999). The nuclease-deficient mutants of Mre11 then fall into two categories: those which are affected for the other roles of Mre11 (resistance to damaging agents) and those that are not (no defect in mating type switching or telomere maintenance). Although their meiotic phenotype is identical, nuclease mutants that fail to form a complex with Rad50 and Xrs2 are more affected in mitotic functions (Furuse et al. 1998, Usui et al. 1998, Lewis et al. 2004, Krogh et al. 2005). In all cases the activity affected in rad50S, sae $2 \Delta$ and the nuclease-deficient mrell cells seems to be required for repairing specific kinds of substrates (Rattray et al. 2001), processing ('cleaning') certain types of DNA ends, such as hairpins formed in inverted repeats (Lobachev et al. 2002), flap structures unprocessed in the absence of $\operatorname{Rad} 27$ (Debrauwere et al. 2001, Moreau et al. 2001), or covalent topoisomerase I-DNA complexes after cell treatment with camptothecin (Deng et al. 2005). The meiotic phenotypes of $s a e 2 \Delta$ and rad50S would suggest that these cells should be hypersensitive to trapped topoisomerase II breaks, which accumulate $5^{\prime}$ covalently linked topoisomerase II at their $5^{\prime}$ ends, similar to Spo11. However, topoisomerase II-linked oligonucleotides, that are observed in vegetative cells and probably reflect spontaneous topoisomerase II-induced DSB, are also observed in sae2s or rad50S mutants (Neale et al. 2005). This suggests that the Mre 11 complex and Sae2 are not strictly required for the endonucleolytic processing of these topoisomerase II breaks.
Finally, the Mre11 complex may have a role during resection after the initial endonucleolytic removal of Spo11-linked oligonucleotide. However, studies of mrell mutant alleles on HO-endonuclease induced DSB in vegetative cells indicate that this function is redundant with at least one other protein (Llorente \& Symington 2004). Exo1 is the strongest candidate for being involved in the lengthening of the resection tract from a few bases to hundreds of bases, both in vegetative and in meiotic cells (Moreau et al. 2001, Tsubouchi \& Ogawa 2000).

In addition to meiotic DSB formation and the nuclease activity, another aspect of the Mre11 complex function that is important in meiosis is sensing of DSB and checkpoint activation. This is difficult to separate from the biochemical activity of Mre11, although some mutants have been identified that are deficient in nuclease activity but proficient in checkpoint activation.

Rad50S, sae2s and certain nuclease mutants of MRE11 show delayed meiosis I division, suggesting that a checkpoint is activated (Alani et al. 1990, McKee \& Kleckner 1997, Nairz \& Klein 1997, Prinz et al. 1997). Indeed, the unprocessed DSB that accumulate in rad50S mutants cause a cell cycle delay that is controlled by the Rad53 paralogue, Mre4/Mek1 (Xu et al. 1997). In mammalian cells the Mre11 complex is a sensor of DSB, that activates the Atm protein and is then phosphorylated in response to DNA damage (Stracker et al. 2004). In yeast the Mre11 complex is also involved in checkpoint activation, via the Atm homologue, Tel1 (D'Amours \& Jackson 2001, Usui et al. 2001). In sae2s (or rad50S) mecl double mutants, mitotic cells become resistant to MMS. This is because, in these cells, a checkpoint is permanently activated, probably because some ends are unprocessed. This checkpoint requires both Mre11 and Tel1, and its activation is accompanied by Mre11 and Xrs2 phosphorylation (Usui et al. 2001). In meiotic cells the same checkpoint is activated upon unprocessed DSB accumulation in $s a e 2 \Delta$ or rad50S cells, requiring a 'structurally functional' Mre11 complex and Tel1 (Usui et al. 2001). In meiotic cells accumulating unprocessed DSB, Mre11 accumulates at DSB, as seen by chromatin immunoprecipitation or cytologically as foci (Usui et al. 1998, Borde et al. 2004), which also requires a complex formation but is independent of checkpoint activation (Usui et al. 2001). The Mre11 complex is required for Tell 
activation and autophosphorylation (Nakada et al. 2003). When the Tell checkpoint is activated, the Sae2 protein becomes phosphorylated, and then dephosphorylated as recombination proceeds (Cartagena-Lirola et al. 2006) (Figure 2). Both Tel1 and Mec1 play redundant roles for Sae2 phosphorylation. Mutation of the preferred canonical motifs for phosphorylation by ATM/ATR kinases completely abolished Sae2 phosphorylation during meiosis, and resulted in the accumulation of unprocessed DSB (Cartagena-Lirola et al. 2006). Moreover, deletion of both TELI and MECI also induced the accumulation of unprocessed DSB. Thus, Tel1/ Mec1-mediated Sae2 phosphorylation is probably necessary to activate the nuclease activity of Mre11, probably through a transient interaction between phosphorylated Sae2 and Rad50 (Figure 2). However, this has not been formally proven, since it was not investigated whether the unresected ends formed in tel1 mecl double mutants still have Spo11 attached. Sae2 phosphorylation may also be necessary to switch the checkpoint off by limiting Mre11-dependent recombination checkpoint signalling.

Strikingly, although it is absolutely required for meiotic DSB processing in S. cerevisiae, no homologue of Sae2 has been found in other species, based on sequence similarity. However, there is a functionally equivalent protein in Arabidopsis thaliana, which shows no sequence similarity but whose mutation shows identical meiotic phenotypes as that in S. cerevisiae, including pronounced Spo11 foci, no Rad51 foci and DNA fragmentation from anaphase I

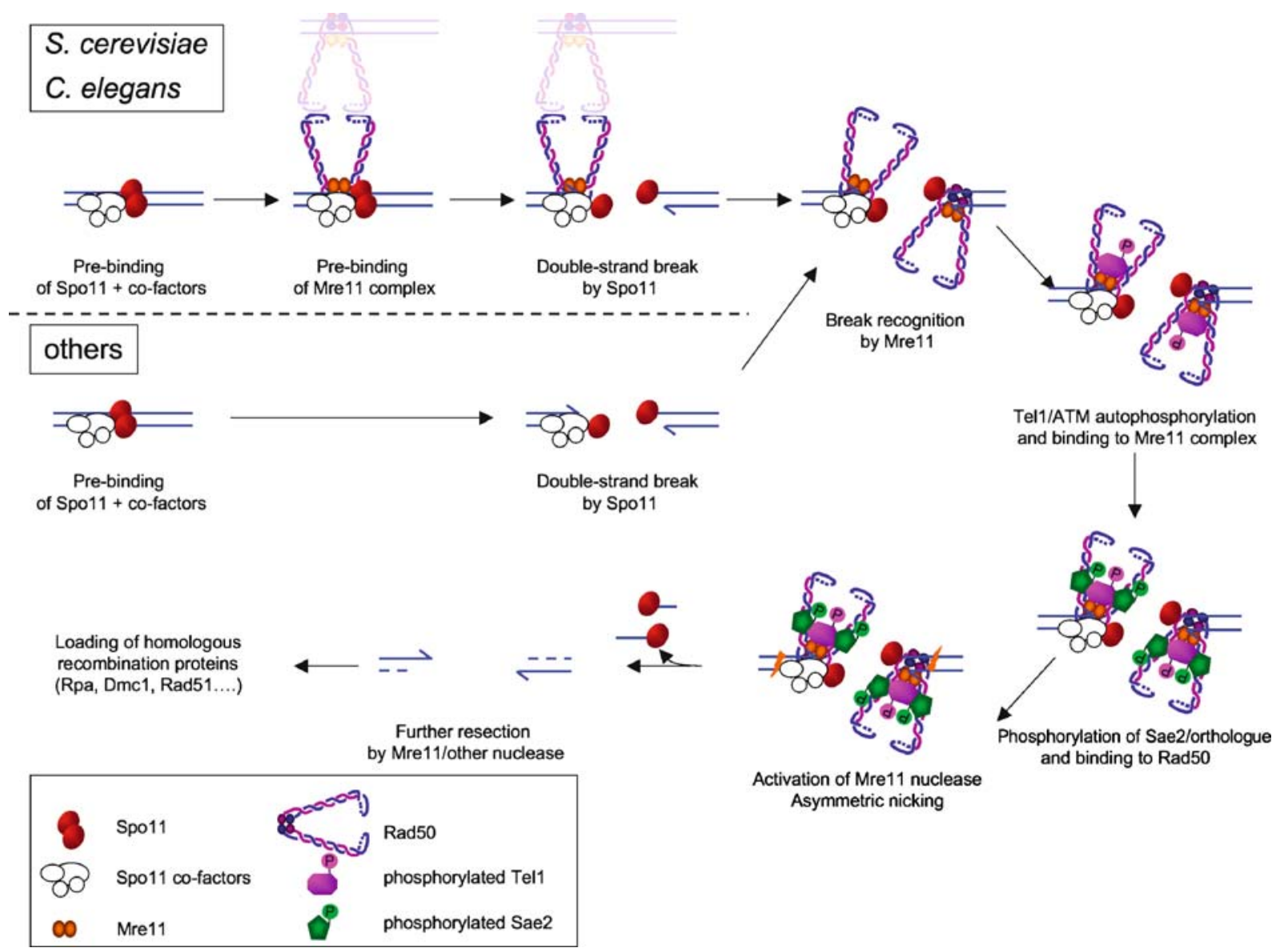

Figure 2. The different steps at which the Mre11 complex is involved during meiotic recombination. For simplicity the Nbs/Xrs2 protein is not drawn. The nature of all the Spo11 'co-factors' is not known for many organisms, but some have been described in S. cerevisiae, S. pombe, A. thaliana and in the mouse. Except in S. cerevisiae and C. elegans, Mre11 seems to be involved only after DSB formation by Spo11 (see text for details). 
onwards (Peter Schloegelhofer, personal communication). This indicates that the function of Sae2 for activating Mre11 nuclease activity during meiotic recombination may be conserved.

\section{Conservation of the roles of the Mre11 complex for meiotic recombination in other organisms}

The phenotype of mutations of the Mre11 complex in vertebrates has long been precluded, since each member of the complex is essential, their deletion leading to embryonic lethality (Stracker et al. 2004). However, the recent discovery of viable hypomorphic mutants, involved in some rare autosomal diseases, has allowed study of the roles of Mre11 complex in different aspects of chromosomes metabolism using mouse models (Stracker et al. 2004). In other eukaryotes the phenotypes of mutations of the Mre11 complex have been described, since the Mre11 complex is not essential. They point to a partial conservation of the meiotic roles defined in S. cerevisiae. In particular, all organisms tested show defects in meiotic recombination in Mre11 complex mutants, e.g. in C. elegans (Chin \& Villeneuve 2001), in Coprinus cinereus (Ramesh \& Zolan 1995, Gerecke \& Zolan 2000, Merino et al. 2000), in Arabidopsis thaliana (Gallego et al. 2001, Bundock \& Hooykaas 2002, Puizina et al. 2004), in Aspergillus nidulans (Semighini et al. 2003, Malavazi et al. 2005) or in Schizzosaccharomyces pombe (Young et al. 2004).

In all organisms studied so far, apart from $C$. elegans and Drosophila, formation and correct processing of homologous recombination intermediates are necessary to promote proper homologous chromosomes synapsis. Thus, cytological observations of meiotic cells allow inferring defects in homologous recombination.

In $C$. elegans, two different mre 11 alleles produced identical phenotypes. One contains a point mutation in a perfectly conserved amino acid $(\mathrm{E} 139 \mathrm{~K})$ in the second phosphoesterase motif and the other one a truncation after amino acid 270 (171 in $S$. cerevisiae), which truncates before the fourth phosphoesterase motif and is predicted to be a null, based on S. cerevisiae studies (Usui et al. 1998). During meiosis both mutants show intact chromosomes, but with no crossing over or chiasmata, suggesting that meiotic recombination did not occur
(Chin \& Villeneuve 2001). Irradiation of mrell germ cells during meiotic prophase induced chromosome fragmentation, indicating a defect in repair of chromosome damage. The fact that, despite their lack of chiasmata, chromosomes in mrell are intact suggests that, in $C$. elegans, Mre11 may, like in $S$. cerevisiae, have a dual role in both generating meiotic DSB and repairing them.

In Coprinus cinereus, Mre11 expression is induced during prophase I of meiosis. An mre11 mutant (mre11-1, truncated after amino acid 315) shows many meiotic defects including incomplete pachytene chromatin condensation, a block in meiotic progression, and defects in chromosome synapsis: some synapsis was observed, but it was not complete and sometimes aberrant synapsis was observed (Gerecke \& Zolan 2000). This is reminiscent of the phenotype observed in mrells, rad50S or sae $2 \Delta$ mutants in yeast, which can form, but cannot process meiotic DSB and show a less severe defect in synapsis than mutants that form no DSB, e.g.

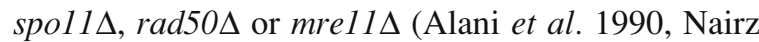
\& Klein 1997, Prinz et al. 1997). Similarly, the Coprinus rad50-4 allele, although strongly defective in synapsis, shows more SC formation than the spo11 mutant, predicted to be completely deficient in DSB formation (Merino et al. 2000). Thus, in Coprinus, the Mre11 complex seems not to be required for meiotic DSB formation, but is probably required for the initiation of their processing.

In the plant Arabidopsis thaliana a truncated mutant of Rad50, lacking the last 266 amino acids, is hypersensitive to MMS and sterile (Gallego et al. 2001). Similarly, a Mre11 mutant (the mre11-1 allele, truncated after amino acid 261, in the phosphoesterase motif IV) is viable, but sterile (Bundock \& Hooykaas 2002). These mutants show no normal pachytene stage and a defect in homologous chromosome pairing and synapsis, and extensive meiotic chromosome fragmentation (Bleuyard et al. 2004, Puizina et al. 2004), which is not seen in a spo11 mutant (Grelon et al. 2001). In addition, the fact that this fragmentation in mre11 mutant is Spo11-dependent clearly suggests that DSB are formed by Spo11 in the Arabidopsis Mre11 complex mutants, but that they are not repaired (Puizina et al. 2004). Finally, the mre11-2 mutant, in which the last 191 amino acids of the Mre11 protein are deleted, is fertile (Bundock \& Hooykaas 2002). Thus, unlike in S. cerevisiae, no 
meiotic function is associated with the C-terminal part of Mre11 in plants.

Finally, in the fission yeast $S$. pombe, Rad50 and Rad32 (the homologue of Mre11) appear not to be required for meiotic DSB formation, although break frequency is reduced. However, both are strictly required for DSB processing, as monitored physically and genetically, the very low spore viability being suppressed by mutations blocking DSB formation (Young et al. 2004).

In conclusion, in all organisms where the effects of mutations of the Mre11 complex could be examined, the complex is required for meiotic recombination, most probably for processing the Spo11-generated DSB. In addition, in $S$. cerevisiae and maybe $C$. elegans, the Mre 11 complex is also required for DSB formation, through a structural rather than catalytical mechanism.

\section{Animal models for mutations in the Mre11 complex}

The embryonic lethality of the disruption of any member of the Mre11 complex in mammals has precluded the study of null mutations (Stracker et al. 2004). However, the discovery that some hypomorphic mutations of Mre11 and Nbs1 are responsible for human genetic instability disorders has allowed the development of mouse models of Mre11 complex deficiency. Three genetic disorders, A-T (mutated in the checkpoint ATM gene), ATLD (mutated in MRE11 gene) and NBS (mutated in NBS1) show patients sharing common cellular features, such as hypersensitivity to ionizing radiation, radioresistant DNA synthesis (RDS) and abrogation of ATM-dependent events (Stracker et al. 2004). Clinical phenotypes of A-T and NBS are quite distinct but the cellular phenotypes are similar; A-T and ATLD share both clinical and cellular phenotypes. A-T patients are sterile. In Atm-1- mice mutant spermatocytes have chromosome synapsis defects and undergo apoptosis during meiotic prophase I, and ovaries in Atm-/- are devoid of oocytes (Xu et al. 1996), which are eliminated in response to the presence of persistent DSB (Di Giacomo et al. 2005). ATLD is due to either one of three known mutations in the MRE11 gene: one truncation at $\mathrm{R} 633 \mathrm{Z}$, removing the last 75 amino acids, one mutation at N117S, an invariant amino acid located between phosphoesterase motifs III and IV (Stewart et al. 1999) or the W210C mutation, mutating an invariant amino acid just before phosphoesterase motif IV (Fernet et al. 2005) (Figure 1). In the first two cases, patients show strongly reduced levels of the three members of the Mre11 complex, whereas in the third only Nbs1 is strongly reduced. Fertility is not reported for these patients. Both R633Z and N117S mutations have been modelled in S. cerevisiae (Q623Z and N113S mutations, respectively). The C-terminal truncation mutant showed wild-type resistance to irradiation and produced only inviable spores, as expected for a C-terminal truncation that makes no meiotic DSB; the N113S mutation conferred moderate IR sensitivity, and produced viable spores, but to a reduced frequency, which may reflect a correct DSB formation but a partial defect in Mre11 nuclease activity (Stewart et al. 1999). A mouse model reproduced the C-terminal truncated allele (R633Z). Mice recapitulated all cellular features of ATLD patients, including radiation sensitivity, intra-S checkpoint failure and chromosome instability (Theunissen et al. 2003). However, no strong meiotic defect was observed in these mice, apart from a slower progression in meiotic prophase and a higher proportion of incompletely synapsed bivalents (Cherry et al. 2007). Female mice were subfertile, showing high levels of embryonic lethality, but meiotic recombination was not defective, showing the presence of chiasmata. Thus, the C-terminal region of mammalian Mre11, required in $S$. cerevisiae for meiotic DSB formation, is clearly not required for either formation or repair of meiotic DSB in mice. The fact that ATLD mice were fertile suggests two alternative hypotheses: either the reduced amounts of the mutated Mre11 protein in the cells impair some of its mitotic functions, but its nuclease activity is intact and still sufficient to remove Spo11 from meiotic DSBs; or the catalytic nuclease activity of Mre11 is not required for meiotic recombination in mammals. The first hypothesis is probably true, because Spo11-oligonucleotide complexes were seen in mice (Neale et al. 2005), and because the modelled N113S mutation in yeast showed at least a partial DSB repair activity, which requires the nuclease activity (Stewart et al. 1999).

NBS patients show impaired development of gonads and ovarian failure for female patients; $95 \%$ of cases are due to the $657 \Delta 5$ allele, producing a $\mathrm{N}$-terminally truncated protein, the Nbs1 p70 protein, 
expressed through an alternative translation site (Figure 1). Two mice models of NBS have been developed, producing reduced amounts of a $\mathrm{N}$-terminal truncated Nbs1 protein equivalent to the human p70 (Kang et al. 2002, Williams et al. 2002). These models recapitulated most of the cellular phenotypes of NBS patients. In both studies spermatogenesis was normal and no severe meiotic defects were detected, and consistently NBS male mice were fertile. In the first study the females were sterile and showed oogenesis failure, 8-week-old mice ovaries completely lacking any oocytes and follicles (Kang et al. 2002). In the second NBS model both males and females were fertile (Williams et al. 2002). Maybe the discrepancies between these two outcomes come form the difference in the alleles produced. Finally, the meiotic phenotypes associated with Nbs1 dysfunction were studied in more detail in null Nbs1 knockout mice complemented with various alleles of the human NBS1 gene (Difilippantonio et al. 2005). The main allele of NBS $\left(\mathrm{hNbs} 1^{657 \Delta 5}\right)$ rescued the lethality of Nbs1-/mice. Males were fertile although showing a delayed appearance of germ cells. Females were sterile, the adult ovaries being devoid of oocytes. However, meiosis was initiated, and probably meiotic DSB were formed and repaired, since chromosomes were fully synapsed, but the oocytes were blocked in pachytene (Difilippantonio et al. 2005). Thus, the NBS1 allele is blocked in meiotic progression, but probably not in meiotic recombination. It is possible that the N-terminal truncated NBS1 could provide enough of the NBS1 activity required to allow normal spermatogenesis, since from $S$. cerevisiae studies of Xrs2, the region deleted in NBS patients is not required for meiotic recombination (Shima et al. 2005, Tsukamoto et al. 2005). It is also possible that the essential role of ATM in meiosis is not dependent on NBS1 since ATM activates a number of other DNA repair pathways upon DNA strand-break damage. In this context, Brca1, which is also activated by ATM through phosphorylation after DNA strand-break damage, is required for homologous recombination and thus might mediate ATM-dependent functions during meiosis (Cortez et al. 1999, Moynahan et al. 1999, Li et al. 2000).

No mutations in the human RAD50 gene, which is also essential for viability, have been described. A hypomorphic 'Rad50S' mutation has been modelled in the mouse. In the original report concerning budding yeast rad50S alleles, several point mutants of $R A D 50$, all occurring in the $\mathrm{N}$ terminal portion of Rad50 containing the ATP binding domain, were called ' $S$ ' for separation of function, because they showed almost no mitotic phenotype but a complete absence of meiotic DSB repair (Alani et al. 1990). Two of the three Rad50S alleles tested (K6E and R83I, equivalent to yeast K6E and K81I, respectively) were embryonic lethal. Thus, the Rad50K22M allele (equivalent to yeast R20M) was studied (Figure 1). The 'Rad50S' mice showed not much meiotic phenotype, and were fertile: although there was a cellular attrition in testes, there was no meiotic recombination defect, normal meiotic progression and chromosome morphology (Bender et al. 2002). Amazingly, these mice showed almost no cellular phenotype, unlike the other Mre11 complex hypomorphic mutations: cells were not sensitive to DNA damage and showed no radioresistant DNA synthesis (RDS). There were wild-type levels of protein, but a profound impact on the organismal level (Bender et al. 2002). In these mutants there is a constitutive damage (increase of chromosome breakage and constitutive histone H2AX phosphorylation) (Bender et al. 2002) and a constitutive activation of PIPK3 kinases, probably due to unprocessed 'physiological' damage (Bender et al. 2002, Morales et al. 2005). This aspect is reminiscent of the phenotype in yeast, where in $\mathrm{mecl}$ cells, rad50S or sae $2 \Delta$ mutation produces constitutive Tel1 phosphorylation (Usui et al. 2001). Since Rad50 is so well conserved, the enzymatic functions impaired by the murine $S$ mutations are probably the same as the corresponding $S$. cerevisiae mutations. The fact that the Rad50S mice are fertile is puzzling. However, a subtle defect in meiotic recombination might not have been detected in the study (Bender et al. 2002). Maybe the K22M activity is sufficient to allow some Mre11 nuclease activity for removing Spo11 from meiotic breaks, whereas the other two $\mathrm{S}$ alleles tested were too strongly affected in resection of a diverse spectrum of substrates to be viable. In support of a conservation of the effect of the 'canonical' Rad50S mutation is the fact that, when modelled in the fission yeast Schizosaccharomyces pombe, the K81I rad50S mutation was viable and behaved as in S. cerevisiae, showing formation but no resection of meiotic DSB, 
and thus no viable spores (Young et al. 2002). Since Spo11-oligonucleotide complexes, most probably resulting from endonucleolytic release by the Mre11 complex, have been detected during mouse meiosis (Neale et al. 2005), it would be interesting to see if this intermediate exists in the same amounts in the meiotic cells of the different Mre11 complex mutations mouse models, or if there might be an alternative way of processing meiotic DSB in mice.

As a conclusion, the Mre11 complex plays essential roles during meiosis, in signalling meiotic DSB and activating the Tel1/Atm checkpoint, and also a unique catalytical role in removing the Spo11 protein from break ends to allow their resection and repair by homologous recombination. The different viable mouse models used so far to model Mre11 complex deficiencies have not shown a clear meiotic recombination defect. However, it is only possible to use hypomorphic mutants, and it is very likely that the catalytical nuclease function of the Mre11 complex, used for Spo11 removal, is essential for embryonic development and cell viability in mammals. This could be related to the involvement of the nuclease activity of Mre11 in preventing the generation of chromosome inverted duplications due to failures in processing hairpins and in preventing amplification of palindromes (Rattray et al. 2001, Lobachev et al. 2002). Meiosis-specific knockout of the Mre 11 complex should allow one to study specifically the meiotic roles of this complex in mammals.

\section{Acknowledgements}

I thank Alain Nicolas for support, Scott Keeney and Peter Schloegelhofer for personal communications, and Marc-Henri Stern for discussions and helpful comments on the manuscript.

\section{References}

Ajimura M, Leem SH, Ogawa H (1993) Identification of new genes required for meiotic recombination in Saccharomyces cerevisiae. Genetics 133: 51-66.

Alani E, Padmore R, Kleckner N (1990) Analysis of wild-type and rad50 mutants of yeast suggests an intimate relationship between meiotic chromosome synapsis and recombination. Cell 61: 419-436.

Arora C, Kee K, Maleki S, Keeney S (2004) Antiviral protein Ski8 is a direct partner of Spo11 in meiotic DNA break formation, independent of its cytoplasmic role in RNA metabolism. Mol Cell 13: 549-559.

Bender CF, Sikes ML, Sullivan R et al. (2002) Cancer predisposition and hematopoietic failure in $\operatorname{Rad} 50(\mathrm{~S} / \mathrm{S})$ mice. Genes Dev 16: $2237-2251$.

Bhaskara V, Dupre A, Lengsfeld B et al. (2007) Rad50 adenylate kinase activity regulates DNA tethering by Mre11/Rad50 complexes. Mol Cell 25: 647-661.

Blat Y, Protacio RU, Hunter N, Kleckner N (2002) Physical and functional interactions among basic chromosome organizational features govern early steps of meiotic chiasma formation. Cell 111: 791-802.

Bleuyard JY, Gallego ME, White CI (2004) Meiotic defects in the Arabidopsis rad50 mutant point to conservation of the MRX complex function in early stages of meiotic recombination. Chromosoma 113: 197-203.

Borde V, Lin W, Novikov E, Petrini JH, Lichten M, Nicolas A (2004) Association of Mre11p with double-strand break sites during yeast meiosis. Mol Cell 13: 389-401.

Bressan DA, Baxter BK, Petrini JH (1999) The Mre11-Rad50Xrs2 protein complex facilitates homologous recombinationbased double-strand break repair in Saccharomyces cerevisiae. Mol Cell Biol 19: 7681-7687.

Bundock P, Hooykaas P (2002) Severe developmental defects, hypersensitivity to DNA-damaging agents, and lengthened telomeres in Arabidopsis MRE11 mutants. Plant Cell 14: 2451-2462.

Carney JP, Maser RS, Olivares H et al. (1998) The hMre11/ hRad50 protein complex and Nijmegen breakage syndrome: linkage of double-strand break repair to the cellular DNA damage response. Cell 93: 477-486.

Cartagena-Lirola H, Guerini I, Viscardi V, Lucchini G, Longhese MP (2006) Budding yeast Sae2 is an in vivo target of the Mec1 and Tel1 checkpoint kinases during meiosis. Cell Cycle 5: 1549-1559.

Cherry SM, Adelman CA, Theunissen JW, Hassold TJ, Hunt PA, Petrini JH (2007) The Mre11 complex influences DNA repair, synapsis, and crossing over in murine meiosis. Curr Biol 17: 373-378.

Chin GM, Villeneuve, AM (2001) C. elegans mre-11 is required for meiotic recombination and DNA repair but is dispensable for the meiotic G(2) DNA damage checkpoint. Genes Dev 15: 522-534.

Cortez D, Wang Y, Qin J, Elledge SJ (1999) Requirement of ATM-dependent phosphorylation of brcal in the DNA damage response to double-strand breaks. Science 286: 1162-1166.

D'Amours D, Jackson SP (2001) The yeast Xrs2 complex functions in $\mathrm{S}$ phase checkpoint regulation. Genes Dev 15: 2238-2249.

D'Amours D, Jackson SP (2002) The Mre11 complex: at the crossroads of DNA repair and checkpoint signalling. Nat Rev Mol Cell Biol 3: 317-327.

de Jager M, van Noort J, van Gent DC, Dekker C, Kanaar R, Wyman C (2001) Human Rad50/Mre11 is a flexible complex that can tether DNA ends. Mol Cell 8: 1129-1135.

Debrauwere H, Loeillet S, Lin W, Lopes J, Nicolas A (2001) Links between replication and recombination in Saccharomyces cerevisiae: a hypersensitive requirement for homologous recombination in the absence of Rad27 activity. Proc Natl Acad Sci USA 98: 8263-8269. 
Deng C, Brown JA, You D, Brown JM (2005) Multiple endonucleases function to repair covalent topoisomerase I complexes in Saccharomyces cerevisiae. Genetics 170: 591-600.

Desai-Mehta A, Cerosaletti KM, Concannon P (2001) Distinct functional domains of nibrin mediate Mre11 binding, focus formation, and nuclear localization. Mol Cell Biol 21: 2184-2191.

Di Giacomo M, Barchi M, Baudat F, Edelmann W, Keeney S, Jasin M (2005) Distinct DNA-damage-dependent and -independent responses drive the loss of oocytes in recombination-defective mouse mutants. Proc Natl Acad Sci USA 102: 737-742.

Difilippantonio S, Celeste A, Fernandez-Capetillo O et al. (2005) Role of Nbs1 in the activation of the Atm kinase revealed in humanized mouse models. Nat Cell Biol 7: 675-685.

Fernet M, Gribaa M, Salih MA, Seidahmed MZ, Hall J, Koenig M (2005) Identification and functional consequences of a novel MRE11 mutation affecting 10 Saudi Arabian patients with the ataxia telangiectasia-like disorder. Hum Mol Genet 14: 307-318.

Furuse M, Nagase Y, Tsubouchi H, Murakami-Murofushi K, Shibata T, Ohta K (1998) Distinct roles of two separable in vitro activities of yeast Mre11 in mitotic and meiotic recombination. EMBO J 17: 6412-6425.

Gallego ME, Jeanneau M, Granier F, Bouchez D, Bechtold N, White CI (2001) Disruption of the Arabidopsis RAD50 gene leads to plant sterility and MMS sensitivity. Plant J 25: 31-41.

Gerecke EE, Zolan ME (2000) An mre11 mutant of Coprinus cinereus has defects in meiotic chromosome pairing, condensation and synapsis. Genetics 154: 1125-1139.

Grelon M, Vezon D, Gendrot G, Pelletier G (2001) AtSPO11-1 is necessary for efficient meiotic recombination in plants. Embo $J$ 20: 589-600.

Hopfner KP, Craig L, Moncalian G et al. (2002) The Rad50 zinchook is a structure joining Mre11 complexes in DNA recombination and repair. Nature 418: 562-566.

Hopfner KP, Karcher A, Shin DS et al. (2000) Structural biology of Rad50 ATPase: ATP-driven conformational control in DNA double-strand break repair and the ABC-ATPase superfamily. Cell 101: 789-800.

Ivanov EL, Korolev VG, Fabre F (1992) XRS2, a DNA repair gene of Saccharomyces cerevisiae, is needed for meiotic recombination. Genetics 132: 651-664.

Johzuka K, Ogawa H (1995) Interaction of Mre11 and Rad50: two proteins required for DNA repair and meiosis-specific doublestrand break formation in Saccharomyces cerevisiae. Genetics 139: $1521-1532$.

Kang J, Bronson RT, Xu Y (2002) Targeted disruption of NBS1 reveals its roles in mouse development and DNA repair. Embo $J$ 21: $1447-1455$.

Keeney S (2001) Mechanism and control of meiotic recombination initiation. Curr Top Dev Biol 52: 1-53.

Keeney S, Giroux CN, Kleckner N (1997) Meiosis-specific DNA double-strand breaks are catalyzed by Spo11, a member of a widely conserved protein family. Cell 88: 375-384.

Kobayashi J, Antoccia A, Tauchi H, Matsuura S, Komatsu K (2004) NBS1 and its functional role in the DNA damage response. DNA Repair (Amst) 3: 855-861.
Kobayashi J, Tauchi H, Sakamoto S et al. (2002) NBS1 localizes to gamma-H2AX foci through interaction with the FHA/BRCT domain. Curr Biol 12: 1846-1851.

Krogh BO, Llorente B, Lam A, Symington LS (2005) Mutations in Mre11 phosphoesterase motif I that impair Saccharomyces cerevisiae Mre11-Rad50-Xrs2 complex stability in addition to nuclease activity. Genetics 171: 1561-1570.

Lee JH, Paull TT (2004) Direct activation of the ATM protein kinase by the Mre11/Rad50/Nbs1 complex. Science 304: 93-96.

Lewis LK, Storici F, Van Komen S, Calero S, Sung P, Resnick MA (2004) Role of the nuclease activity of Saccharomyces cerevisiae Mre11 in repair of DNA double-strand breaks in mitotic cells. Genetics 166: 1701-1713.

Li S, Ting NS, Zheng L, et al. (2000) Functional link of BRCA1 and ataxia telangiectasia gene product in DNA damage response. Nature 406: 210-215.

Llorente B, Symington LS (2004) The Mre11 nuclease is not required for $5^{\prime}$ to $3^{\prime}$ resection at multiple $\mathrm{HO}$-induced doublestrand breaks. Mol Cell Biol 24: 9682-9694.

Lobachev KS, Gordenin DA, Resnick MA (2002) The Mre11 complex is required for repair of hairpin-capped double-strand breaks and prevention of chromosome rearrangements. Cell 108: 183-193.

Malavazi I, Lima JF, von Zeska Kress Fagundes et al. (2005) The Aspergillus nidulans sldI(RAD50) gene interacts with bimE(APC1), a homologue of an anaphase-promoting complex subunit. Mol Microbiol 57: 222-237.

McKee AH, Kleckner N (1997) A general method for identifying recessive diploid-specific mutations in Saccharomyces cerevisiae, its application to the isolation of mutants blocked at intermediate stages of meiotic prophase and characterization of a new gene SAE2. Genetics 146: 797-816.

Merino ST, Cummings WJ, Acharya SN, Zolan ME (2000) Replication-dependent early meiotic requirement for Spo11 and Rad50. Proc Natl Acad Sci USA 97: 10477-10482.

Morales M, Theunissen JW, Kim CF, Kitagawa R, Kastan MB, Petrini JH (2005) The Rad50S allele promotes ATM-dependent DNA damage responses and suppresses ATM deficiency: implications for the Mre11 complex as a DNA damage sensor. Genes Dev 19: 3043-3054.

Moreau S, Ferguson JR, Symington LS (1999) The nuclease activity of Mre11 is required for meiosis but not for mating type switching, end joining, or telomere maintenance. Mol Cell Biol 19: $556-566$.

Moreau S, Morgan EA, Symington LS (2001) Overlapping functions of the Saccharomyces cerevisiae Mre11, Exo1 and $\operatorname{Rad} 27$ nucleases in DNA metabolism. Genetics 159: 1423-1433.

Moreno-Herrero F, de Jager M, Dekker NH, Kanaar R, Wyman C, Dekker C (2005) Mesoscale conformational changes in the DNA-repair complex Rad50/Mre11/Nbs1 upon binding DNA. Nature 437: 440-443.

Moynahan ME, Chiu JW, Koller BH, Jasin M (1999) Brca1 controls homology-directed DNA repair. Mol Cell 4: 511-518.

Nairz K, Klein F (1997) mre11S-a yeast mutation that blocks double-strand-break processing and permits nonhomologous synapsis in meiosis. Genes Dev 11: 2272-2290. 
Nakada D, Matsumoto K, Sugimoto K (2003) ATM-related Tel1 associates with double-strand breaks through an Xrs2-dependent mechanism. Genes Dev 17: 1957-1962.

Neale MJ, Pan J, Keeney S (2005) Endonucleolytic processing of covalent protein-linked DNA double-strand breaks. Nature 436: 1053-1057.

Paull TT, Gellert M (1998) The $3^{\prime}$ to $5^{\prime}$ exonuclease activity of Mre 11 facilitates repair of DNA double-strand breaks. Mol Cell 1: 969-979.

Paull TT, Gellert M (1999) Nbs1 potentiates ATP-driven DNA unwinding and endonuclease cleavage by the Mre11/Rad50 complex. Genes Dev 13: 1276-1288.

Prinz S, Amon A, Klein F (1997) Isolation of COM1, a new gene required to complete meiotic double-strand break-induced recombination in Saccharomyces cerevisiae. Genetics 146: 781-795.

Puizina J, Siroky J, Mokros P, Schweizer D, Riha K (2004) Mre11 deficiency in Arabidopsis is associated with chromosomal instability in somatic cells and Spo11-dependent genome fragmentation during meiosis. Plant Cell 16: 1968-1978.

Ramesh MA, Zolan ME (1995) Chromosome dynamics in rad12 mutants of Coprinus cinereus. Chromosoma 104: 189-202.

Rattray AJ, McGill CB, Shafer BK, Strathern JN (2001) Fidelity of mitotic double-strand-break repair in Saccharomyces cerevisiae: a role for SAE2/COM1. Genetics 158: 109-122.

Semighini CP, von Zeska Kress Fagundes MR, Ferreira JC et al. (2003) Different roles of the Mre11 complex in the DNA damage response in Aspergillus nidulans. Mol Microbiol 48: 1693-1709.

Shima H, Suzuki M, Shinohara M (2005) Isolation and characterization of novel xrs2 mutations in Saccharomyces cerevisiae. Genetics 170: 71-85.

Stewart GS, Maser RS, Stankovic T et al. (1999) The DNA double-strand break repair gene hMRE11 is mutated in individuals with an ataxia-telangiectasia-like disorder. Cell 99: 577-587.

Stracker TH, Theunissen JW, Morales M, Petrini JH (2004) The Mre11 complex and the metabolism of chromosome breaks: the importance of communicating and holding things together. DNA Repair (Amst) 3: 845-854.

Takata H, Tanaka Y, Matsuura A (2005) Late S phase-specific recruitment of Mre11 complex triggers hierarchical assembly of telomere replication proteins in Saccharomyces cerevisiae. Mol Cell 17: 573-583.

Tauchi H, Kobayashi J, Morishima K et al. (2001) The forkhead-associated domain of NBS1 is essential for nuclear foci formation after irradiation but not essential for hRAD50hMRE11-NBS1 complex DNA repair activity. J Biol Chem 276: $12-15$.
Theunissen JW, Kaplan MI, Hunt PA et al. (2003) Checkpoint failure and chromosomal instability without lymphomagenesis in Mre11(ATLD1/ATLD1) mice. Mol Cell 12: 1511-1523.

Trujillo KM, Roh DH, Chen L, Van Komen S, Tomkinson A, Sung P (2003) Yeast xrs2 binds DNA and helps target rad50 and mre11 to DNA ends. J Biol Chem 278: 48957-48964.

Tsubouchi H, Ogawa H (1998) A novel mre11 mutation impairs processing of double-strand breaks of DNA during both mitosis and meiosis. Mol Cell Biol 18: 260-268.

Tsubouchi H, Ogawa H (2000) Exo1 roles for repair of DNA double-strand breaks and meiotic crossing over in Saccharomyces cerevisiae. Mol Biol Cell 11: 2221-2233.

Tsukamoto Y, Mitsuoka C, Terasawa M, Ogawa H, Ogawa T (2005) Xrs2p regulates Mre11p translocation to the nucleus and plays a role in telomere elongation and meiotic recombination. Mol Biol Cell 16: 597-608.

Usui T, Ogawa H, Petrini JH (2001) A DNA damage response pathway controlled by Tel1 and the Mre11 complex. Mol Cell 7: 1255-1266.

Usui T, Ohta T, Oshiumi H, Tomizawa J, Ogawa H, Ogawa T (1998) Complex formation and functional versatility of Mre11 of budding yeast in recombination. Cell 95: 705-716.

Uziel T, Lerenthal Y, Moyal L, Andegeko Y, Mittelman L, Shiloh Y (2003) Requirement of the MRN complex for ATM activation by DNA damage. Embo $J$ 22: 5612-5621.

Williams BR, Mirzoeva OK, Morgan WF, Lin J, Dunnick W, Petrini JH (2002) A murine model of Nijmegen breakage syndrome. Curr Biol 12: 648-653.

Wiltzius JJ, Hohl M, Fleming JC, Petrini JH (2005) The Rad50 hook domain is a critical determinant of Mre11 complex functions. Nat Struct Mol Biol 12: 403-407.

Xu L, Weiner BM, Kleckner N (1997) Meiotic cells monitor the status of the interhomolog recombination complex. Genes Dev 11: $106-118$.

Xu Y, Ashley T, Brainerd EE, Bronson RT, Meyn MS, Baltimore D (1996) Targeted disruption of ATM leads to growth retardation, chromosomal fragmentation during meiosis, immune defects, and thymic lymphoma. Genes Dev 10: 2411-2422.

You Z, Chahwan C, Bailis J, Hunter T, Russell P (2005) ATM activation and its recruitment to damaged DNA require binding to the C terminus of Nbs1. Mol Cell Biol 25: 5363-5379.

Young JA, Hyppa RW, Smith GR (2004) Conserved and nonconserved proteins for meiotic DNA breakage and repair in yeasts. Genetics 167: 593-605.

Young JA, Schreckhise RW, Steiner WW, Smith GR (2002) Meiotic recombination remote from prominent DNA break sites in S. pombe. Mol Cell 9: 253-263.

Zickler D, Kleckner N (1999) Meiotic chromosomes: integrating structure and function. Annu Rev Genet 33: 603-754. 